\title{
Erratum to: BMP9 is produced by hepatocytes and circulates mainly in an active mature form complexed to its prodomain
}

\author{
Marie Bidart • Nicolas Ricard - Sandrine Levet $\cdot$ Michel Samson • \\ Christine Mallet $\cdot$ Laurent David $\cdot$ Mariela Subileau $\cdot$ Emmanuelle Tillet \\ Jean-Jacques Feige $\cdot$ Sabine Bailly
}

Published online: 31 July 2011

(C) Springer Basel AG 2011

\section{Erratum to: Cell Mol Life Sci \\ DOI 10.1007/s00018-011-0751-1}

The following Acknowledgement was missed in original publication of this article.

Acknowledgments This work was supported by INSERM, CEA (Commissariat à l'Energie Atomique), UJF (Université Joseph Fourier, Grenoble) and ARC (Association pour la Recherche sur le Cancer). N.R. was supported by the CEA and the ARC. SL was supported by the CEA.

The online version of the original article can be found under doi:10.1007/s00018-011-0751-1.

M. Bidart - N. Ricard · S. Levet - C. Mallet - L. David .

M. Subileau $\cdot$ E. Tillet $\cdot$ J.-J. Feige $\cdot$ S. Bailly $(\bowtie)$

Unit 1036, Biology of Cancer and Infection, INSERM,

17 rue des Martyrs, 38054 Grenoble, France

e-mail: sbailly@cea.fr

M. Bidart - N. Ricard - S. Levet - C. Mallet - L. David .

M. Subileau $\cdot$ E. Tillet $\cdot$ J.-J. Feige $\cdot$ S. Bailly

UJF-Grenoble 1, Biology of Cancer and Infection,

38041 Grenoble, France

M. Bidart - N. Ricard - S. Levet - C. Mallet - L. David .

M. Subileau $\cdot$ E. Tillet $\cdot$ J.-J. Feige $\cdot$ S. Bailly

CEA, DSV/iRTSV, Biology of Cancer and Infection,

38054 Grenoble, France

M. Bidart

Pôle Recherche, Centre Hospitalier Universitaire de Grenoble,

38043 Grenoble, France

M. Samson

U620/EA 4427 SeRAIC, INSERM, Université Rennes 1,

35043 Rennes, France

L. David

Center for Systems Biology, Samuel Lunenfeld Research

Institute, Mount Sinai Hospital, Toronto, ON M5G 1X5, Canada 